\title{
Correlates of maternal weight and body mass index after childbirth
}

\author{
L. Mullaney ${ }^{1}$, S. Cawley ${ }^{1}$, A. O’Higgins ${ }^{2}$, N. Daly ${ }^{2}$, D. McCartney ${ }^{1}$ and M.J. Turner ${ }^{2}$ \\ ${ }^{1}$ School of Biological Sciences, Dublin Institute of Technology, Dublin 8, Republic of Ireland and ${ }^{2} U C D$ Centre for \\ Human Reproduction, Coombe Women and Infants University Hospital, Dublin 8, Republic of Ireland
}

It has been recommended that obese women should limit weight gain during and after pregnancy. However weight management intervention studies in obese women have in general been unsuccessful. ${ }^{(1,2)}$ The aim of this paper is to investigate diet, physical activity and socioeconomic factors associated with BMI changes from early pregnancy to nine months postpartum, and with postpartum weight status.

Women were recruited at their convenience at the first antenatal visit. Weight was measured in early pregnancy and repeated at four and nine months postpartum. Detailed data were collected on diet and exercise habits, infant feeding practices and socioeconomic status; in addition to routine clinical and sociodemographic parameters.

On univariate analysis, smoking, consistent poverty and $>30$ years of age appeared to be associated with postpartum weight and BMI losses. On multivariate analysis, women who were obese in early pregnancy were more likely to have experienced weight gain at nine months postpartum [OR $2 \cdot 185, P=0 \cdot 04]$. The relationship with smoking and postpartum weight change reversed on multivariate analysis, with smokers now more likely to have gained weight by nine months postpartum [OR 2.388, $P=0 \cdot 03$ ] (Table 1 ).

Table 1. Logistic regression of factors associated with maternal weight gain at nine months postpartum

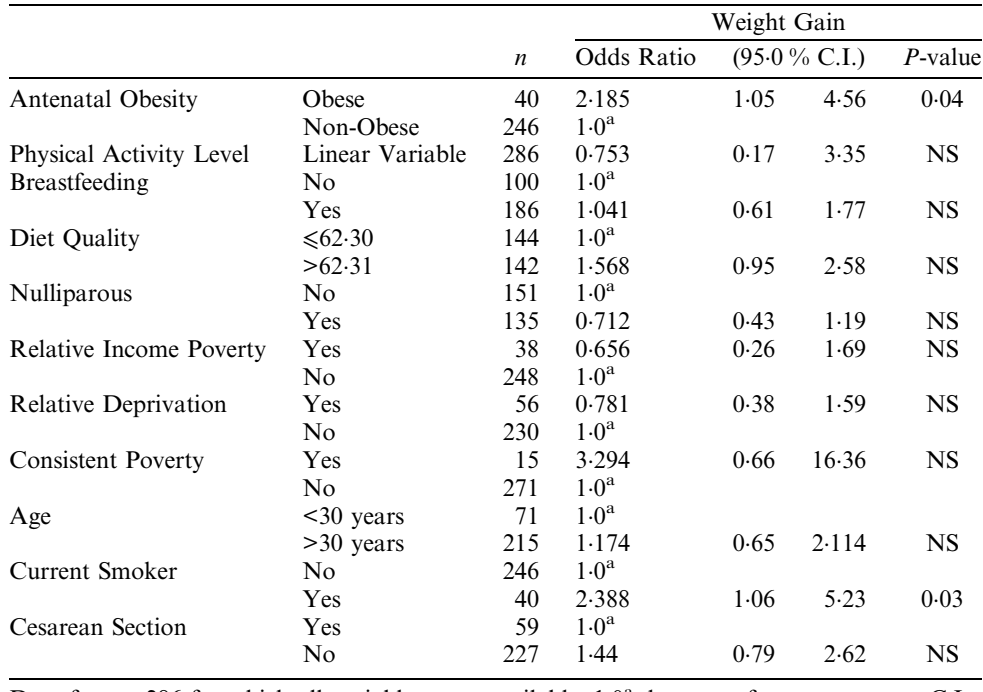

Data for $n=286$ for which all variables were available, $1 \cdot 0^{\text {a }}$ denotes reference category, C.I. confidence interval.

At nine months postpartum, weight gain is associated with maternal obesity and smoking at the first antenatal visit, but not with maternal physical activity, diet or socioeconomic status. This suggests that preconceptional weight management interventions should be specifically aimed at obese smokers.

1. Institute of Medicine. (2009) Washington, DC: The National Academies Press.

2. O'Higgins AC, Doolan A, Mullaney L et al. (2013) J Perinat Med 21,1-7. 